\title{
Aquaporins as diagnostic and therapeutic targets in cancer: How far we are?
}

\author{
Jian Wang ${ }^{1 \dagger}$, Li Feng ${ }^{2 \dagger}$, Zhitu Zhu ${ }^{3+}$, Minghuan Zheng ${ }^{1}$, Diane Wang ${ }^{1}$, Zhihong Chen ${ }^{1 *}$ and Hongzhi Sun ${ }^{3^{*}}$
}

\begin{abstract}
Aquaporins (AQPs) are a family of water channel proteins distributed in various human tissues, responsible for the transport of small solutes such as glycerol, even gas and ions. The expression of AQPs has been found in more than 20 human cancer types and is significantly correlated with the severity of histological tumors and prognosis of patients with cancer. More recent evidence showed that AQPs could also play a role in tumor-associated edema, tumor cell proliferation and migration, and tumor angiogenesis in solid and hematological tumors. Inhibitors of AQPs in tumor cells and microvessels have been suggested as new therapeutic strategies. The present review overviews AQPs structures, expression variation among normal tissues and tumors, AQPs functions and roles in the development of cancer with special focuses on lung, colorectal, liver, brain and breast cancers, and potential AQPs-target inhibitors. We call the special attention to consider AQPs important as diagnostic and therapeutic biomarkers. It may be a novel anticancer therapy by the AQPs inhibition.
\end{abstract}

Keywords: AQPs, Cancer, Water channel, Inhibition, Biomarkers

\section{Introduction}

The aquaporins (AQPs) are a family of small, transmembrane, water-transport proteins distributed in various human tissues [1]. Thirteen members in AQPs family have been identified in humans, divided into two groups according to transported materials. For example, AQP1, AQP2, AQP4, AQP5, or AQP8 are exclusively selective for water, while AQP3, AQP7, AQP9, or AQP10 proposed as aquaglyceroporins can transport water and small neutral solutes such as glycerol [2]. Recently AQPs such as AQP1, AQP4, or AQP5 were found to be permeable for ion and gas flow (e.g. $\mathrm{O}_{2}, \mathrm{CO}_{2}$, or nitric oxide) [3-5]. Most AQPs are located in plasma membrane to drive osmotic-gradients-dependent water transport, while $\mathrm{AQP} 11$ and $\mathrm{AQP} 12$, as the super-aquaporins, are expressed in cytoplasm to regulate intracellular water transport, organelle volume, or intra-vesicular homeostasis [6]. AQP4 has multiple isoforms, although the number of AQP4 isoforms remains uncertain $[7,8]$. AQP4-M1 isoform is the full-length protein with the

\footnotetext{
* Correspondence: chen.zhihong@zs-hospital.sh.cn; cmushz@163.com ${ }^{\dagger}$ Equal contributors

${ }^{1}$ Department of Pulmonary Medicine, Zhongshan Hospital, Fudan University, Shanghai, China

${ }^{3}$ The First Hospital of Liaoning Medical University, Jingzhou, China Full list of author information is available at the end of the article
}

sequence starting at methionine 1, whereas AQP4-M23 isoform starts at methionine 23 without the first 22 amino acid sequences [9]. AQP4-M1 and AQP4-M23 can form supra-molecular structures as orthogonal arrays of particles (OAPs), of which the size is positively associated with M23:M1 ratio [10]. OAPs assemblies were suggested to be required for NMO-IgG to recognize AQP4 in neuromyelitis optica (NMO), although the function of OAPs was still unclear [11].

A large number of studies showed that AQPs were closely associated with cancer biological functions and expressed in more than 20 human cancer cell types [9]. AQPs expression is positively correlated with tumor types, grades, proliferation, migration, angiogenesis, or tumor-associated edema $[2,12,13]$, which can be considered as diagnostic and therapeutic targets in anticancer treatment. For example, AQP1 and AQP4 were massively up-regulated in high-grade astrocytomas, as compared with low-grade ones and normal brain tissues [13,14]. The high expression of AQP4 was correlated with the formation of more prominent brain edema [15]. AQP1 was up-regulated in lung adenocarcinoma and inhibition of AQP1 expression can inhibit tumor cell invasion, which thereby were proposed as the prognostic index and therapeutic target for lung 
cancer [16]. Fortunately, many AQPs-target inhibitors have been developed to damage tumor cells. But further researches were required to verify the efficiency and safety of these AQPs-target inhibitors in clinical therapy. The present review overviews AQPs structures, AQPs expression in normal and tumor tissues, AQPs functions and specific roles in cancer development, and potential AQPs-target anticancer drugs.

\section{AQPs structures}

Each AQP monomer weighs about between 28 and $30 \mathrm{kDa}$ and has six-titled $\alpha$-helical domains to form a barrel-like structure spanning plasma membrane (Figure 1A). The polypeptide in the structure is formed by a single chain with about 270 amino acids, and amino $(\mathrm{N})$ and carboxyl (C) terminals are located in the cytoplasm [17]. Two highly conserved sequence motifs asparagineproline-alanine (NPA) with a short helix are located on opposite sides of monomer. NPA motifs bend into molecule to pair with each other and form the water channel [18]. A cysteine residue (Cys 189) can be found in most members of AQPs family and resides near the channel in extracellular orientation, which can block AQPs with functional sensitivity to mercury [19].

AQP monomer contains independently water pore and assembles as homotetramers to contribute to fluid transport [20] (Figure 1B). As for the narrow pore with the diameter of about $2.8 \AA$ at the narrowest point and
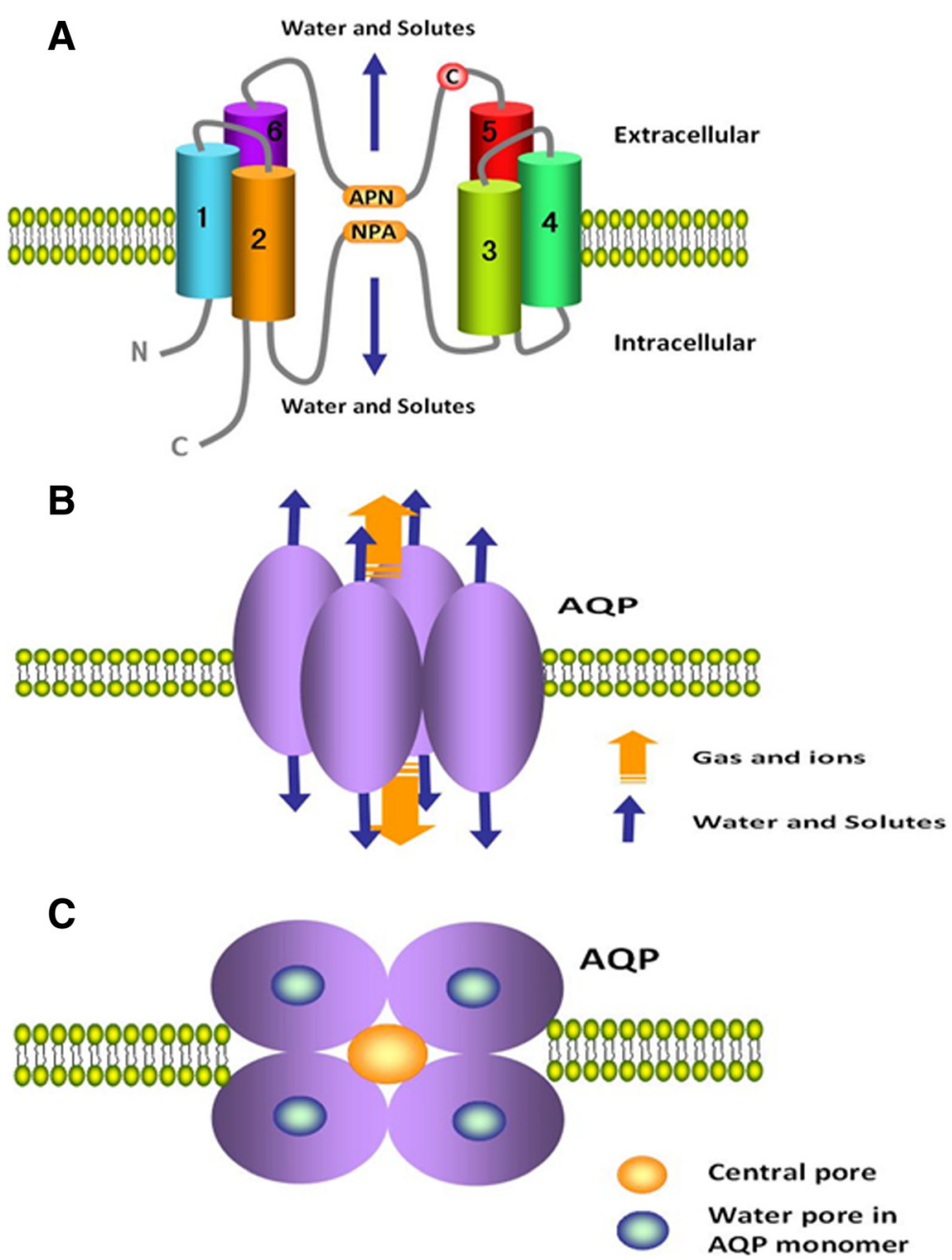

Figure 1 The structures of AQP monomer and homotetramers. (A) Each AQP monomer has six titled a-helical domains to form a water pore spanning plasma membrane. Conserved sequence motifs NPA on the loops bend into molecule to pair with each other and form the water channel. C, in red, represents a cysteine residue (Cys 189) that can block the AQPs function with functional sensitivity to mercury. (B) The structure of AQP homotetramers from side view. Each AQP monomer contains independently a water pore. AQP monomers assemble as homotetramers to form a central pore in homotetramers. Red arrow represents the central pore with transporting gas and ions. Blue arrow represents the water pore with transporting water and solutes. (C) The structure of AQP homotetramers from the top view. Each AQP monomer contains independently a water pore. AQP monomers assemble as homotetramers to form a central pore in homotetramers. 
electrostatic interactions, AQP1, AQP2, AQP4, AQP5, and AQP8 mediate water translocation in single file and block proton transport $[17,21,22]$. This process is dependent on osmotic gradients without ATP expense [17]. As to aquaglyceroporins, AQP3, AQP7, AQP9, and AQP10 contain two additional peptide spans and modulate the size of pore with a diameter of $3.4 \AA$ at the narrowest point) to transport glycerol $[20,23]$.

AQP monomers assembly forms a central pore in homotetramers (Figure 1C). Several studies indicated that the central pore was permeable for gases $\mathrm{O}_{2}, \mathrm{CO}_{2}$, or nitric oxide in AQP1, AQP4, and AQP5 [3-5]. The AQPs-dependent gas transport is faster than free diffusion and plays an important role in the biological function. For example, AQP1-dependent $\mathrm{CO}_{2}$ transport in proximal tubules of the kidney regulates arterial $\mathrm{pH}$ during metabolic acidosis, possibly by acting as a $\mathrm{CO}_{2}$ transporter [24,25]. The ability of aquaporins to transport NO may tightly control $\mathrm{NO}$ concentrations in target cells and directional releases from NO-producing cells mediates $\mathrm{NO}$-dependent relaxation in the vasculature $[26,27]$. However, the molecular mechanism of gas transport through the central pore remains unclear.

\section{AQPs expression in normal and cancer tissues}

Human AQPs distribute in different normal tissue and most of them are located in epithelium and endothelium, as well as some other typical cells such as erythrocytes, astrocytes, adipocytes and skeletal muscle [28]. $\mathrm{AQP1}, \mathrm{AQP} 3, \mathrm{AQP} 8$ and $\mathrm{AQP9}$ have a wide expression in a variety of human tissues and act as special functions in different organs [17]. AQP0 is expressed in human lens, involving in lens transparency and homeostasis [29]. AQP2 and AQP6 are mainly found in kidney and regulate the urinary concentration [30,31]. The significant role of AQP4 is involved with brain edema for its up-expression in stroke, trauma, tumor and other central nervous system (CNS) disorders, although it is normally located in perivascular astrocyte foot processes [32]. AQP5 is mainly expressed in lung, gastrointestinal tract and secretory glands [33], while AQP7 is found in adipocytes, skeletal muscle, heart and kidney [17].

AQPs have been found to express in several solid and hematological cancer cells. The detail of AQPs expression in different cancer tissues is summarized in Table 1. One type of AQP protein can be expressed in different cancer tissues and there is a lack of the tumor-specific prosperity. AQP1 is over-expressed in brain, lung, breast, colorectal, or ovarian cancers [13,34-37]. The expression of AQP3 is up-regulated in cutaneous, esophageal and oral squamous, pulmonary, renal, or hepatocellular cancers [38-42]. The expression of AQP4 is increased in brain, lung, or thyroid cancers $[14,43,44]$. AQP5 is overexpressed in lung, chronic myelogenous leukemia (CML), ovarian, stomach, or colorectal cancers [45-49]. AQP7 expression is increased in thyroid cancer, while AQP9 mainly in brain or ovarian cancers [50-52]. However AQP8 is down-expressed in hepatocellular or colorectal cancers $[53,54]$. In other hands, one type of cancer can also present a close association with several different AQPs. The AQPs in lung, colorectal, liver, brain and breast cancers are showed in Figure 2.

\section{AQPs functions}

The physiological and pathological functions of $A Q P$ proteins have been investigated in the condition of $A Q P$ deletion. In normal human tissues, AQPs regulate brain water homeostasis, exocrine gland secretion, urine concentration, skin moisturization, fat metabolism or neural signal transduction. In the CNS, AQP4 plays a principle role in the regulation of blood-brain barrier (BBB) water permeability while AQP1 facilitates cerebrospinal fluid

Table 1 AQPs expression in different human tumors

\begin{tabular}{|c|c|c|}
\hline Aquaporins & Tumor type & Correlated function \\
\hline AQP1 & $\begin{array}{l}\text { Brain cancer [13], Breast cancer [35], Colorectal cancer [34], Cervical } \\
\text { cancer, Laryngeal cancer, Lung cancer [43], Nasopharyngeal cancer, } \\
\text { Ovarian cancer [37] }\end{array}$ & $\begin{array}{l}\text { Tumor grade, prognosis, tumor angiogenesis, tumor } \\
\text { necrosis, tumor cell migration, tumor invasion and } \\
\text { metastasis. }\end{array}$ \\
\hline AQP3 & $\begin{array}{l}\text { Colorectal cancer [34], Cervical cancer, Liver cancer [40], Lung cancer } \\
\text { [9], Oesophageal cancer [39], Renal cancer [41], Skin cancer [38], } \\
\text { Stomach cancer, Tongue cancer [42] }\end{array}$ & $\begin{array}{l}\text { Tumor grade, prognosis, tumor angiogenesis, tumor } \\
\text { invasion, tumor cell migration and tumor energy } \\
\text { metabolism. }\end{array}$ \\
\hline AQP4 & Brain cancer [14], Lung cancer [43], Thyroid cancer [44] & $\begin{array}{l}\text { Tumor grade, migration, tumor-associated edema, } \\
\text { adhesion, invasion and tumor apoptosis. }\end{array}$ \\
\hline AQP5 & $\begin{array}{l}\text { Breast cancer [9], Colorectal cancer [34,45], Cervical cancer, Leukaemia } \\
\text { [46], Liver cancer [40], Lung cancer [47], Oesophageal cancer [39], } \\
\text { Ovarian cancer [48], Stomach cancer [49], Tongue cancer [42] }\end{array}$ & $\begin{array}{l}\text { Tumor prognosis, tumor proliferation, tumor invasion, } \\
\text { tumor cell migration and drug resistance. }\end{array}$ \\
\hline AQP7 & Thyroid cancer [50] & Unknown \\
\hline AQP8 & Cervical cancer & $\begin{array}{l}\text { Tumor cell migration, tumor invasion, tumor metastasis } \\
\text { and anti-apoptosis. }\end{array}$ \\
\hline AQP9 & Brain cancer [51], Ovarian cancer [52] & Tumor grade, drug resistance and energy metabolism. \\
\hline
\end{tabular}




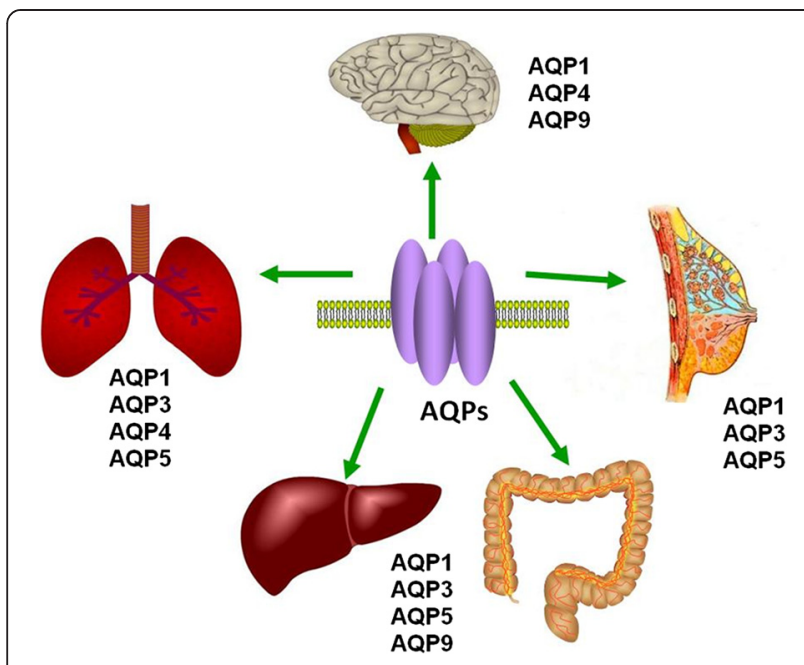

Figure 2 The over-expression of AQPs in cancers as elaborated in the review. The role of AQPs in lung, colorectal, liver, brain and breast cancers has been widely studied.

secretion. The deletion AQP4 can reduce water permeability through the cell plasma membrane in the brain [55-57]. In the salivary glands, AQP5 transports osmotic water into acinar lumen, and regulates the production, viscosity, or tonicity of the saliva [58-60]. Several AQPs (AQP1, AQP2, AQP3, or AQP4) are important in regulating the balance of water in different locations of kidney. The deletion of AQP1 or loss of function mutations in AQP1 could compromise the ability to concentrate the urine maximally during the challenge with water deprivation [61,62]. AQP2 is regulated by vasopressin (AVP) and AQP2 deletion leads to severe polyuria with a low urine osmolality [63]. In normal skin, the basal layer keratinocytes of epidermis express AQP3, involving in water and glycerol transport and skin hydration $[64,65]$. Deletion of AQP3 reduces skin elasticity and skin hydration, reduces biosynthesis of the stratum corneum, or delays wound healing [66]. Besides, AQP7 is suggested to regulate fat metabolism of adipocytes, due to the increased body fat mass, defective glycerol exit, and consequent accumulation of glycerol and triglycerides during AQP7 deletion [67]. The role of AQPs in neural signal transduction is unclear, but AQP4 expression on astroglia is proposed to regulate the volume of the extracellular space, interact with Kir4.1 $\mathrm{K}^{+}$channels, or balance the water re-uptake into astroglia during neural signaling [1].

AQPs also facilitate cell proliferation, migration, and adhesion or angiogenesis in the tissue, which may play an important role in the pathogenesis of cancer. It was reported that vascular endothelial cells expressed AQP1 to play a key role in the pathogenesis of tumor angiogenesis by accelerating cells migration [68]. AQP1-null mice had low ability to form the angiogenesis in the cancer tissue induced by subcutaneous injection of melanoma cells and produced extensive tumor necrosis. Cancer cells with AQP1 over-expression had stronger capacity of cell migration, invasion, and metastasis [69]. AQP1 is thus proposed as a key player in cancer biology and a potential target for drug development. Likewise, AQP4 was strongly expressed in brain and facilitates astroglial cell migration and scarring formation after brain injury, which could be prevented by the deletion of AQP4 $[70,71]$. It was proposed that the water influx at the tip of a lamellipodium through actin cleavage and ion uptake resulted in the membrane protrusion responsible for the AQP-dependent migration [1] (Figure 3). AQP4 was also considered as a critical control factor in cell adhesion in the ocular lens $[72,73]$.

\section{AQPs in lung cancer}

$\mathrm{AQP} 1, \mathrm{AQP} 3, \mathrm{AQP} 4$ and AQP5 are over-expressed in lung cancer $[74,75]$. AQP1 was mainly up-expressed in lung adenocarcinoma (ADC) and bronchoalveolar carcinoma (BAC) instead of lung squamous cell carcinoma and normal lung tissue [16]. The over-expressed AQP1 was located in the endothelial cells of capillaries within lung cancer tissue responsible for the development of angiogenesis $[43,76]$. AQP1 could regulate lung cancer cell invasion and migration which can be inhibited by AQP1-shRNA [43]. The expression of AQP1 was correlated with high postoperative metastasis ratios and low disease-free survival rates in ADCs, especially with micropapillary ADC components [75]. It was proposed that AQP1 could be a significant prognostic index for stage and histologic differentiation of lung cancer.

AQP3 was over-expressed in non-small cell carcinoma (NSCLC), especially adenocarcinomas, or well-differentiated bronchioloalveolar carcinomas and papillary subtypes. It is possible that AQP3 may regulate biological functions of lung cancer cells and be required in the early stage of lung ADC development [77]. AQP3 may be involved in the initiative of angiogenesis in lung cancer through HIF- $2 \alpha$-VEGF pathway, lung cancer cell invasion partly by the AKT-MMPs pathway, cellular glycerol

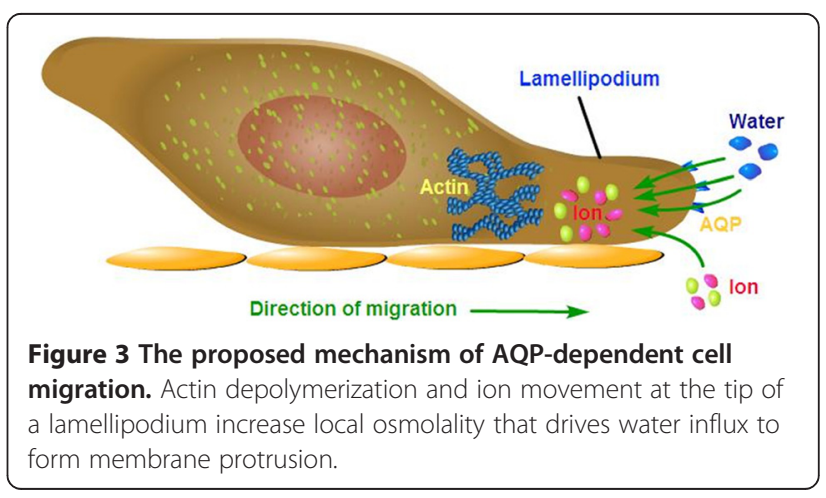


uptake, or mitochondrial ATP formation [78]. The anticancer effects of shRNA-targeting AQP3 were observed in experimental NSCLC, further evidenced by the inhibition of AQP3 deletion for lung cancer growth and prolonged survival in preclinnical studies [78].

AQP5 over-expression was observed predominantly in lung ADCs and associated with poor prognosis of patients with NSCLC. AQP5-positive cells exhibited a loss of epithelial cell markers and activation of c-Src by AQP5 through SH3 binding domain to promote EMT, which might contribute to the enhanced metastasis potential of lung cancer [47]. Over-expressed AQP5 could facilitate lung cancer cell growth and invasion through the activation of the EGFR/ERK/p38 MAPK pathway [79]. The cAMP-protein kinase (PKA) consensus site in AQP5 was preferentially phosphorylated and promoted cell proliferation ability in tumor. The phosphorylation at Ser156 in PKA consensus site was demonstrated as a key role in tumor proliferation and invasion by Ser156 mutants in lung cancer cells [80]. Thus, Ser156 in AQP5 provided a unique opportunity as therapeutic target. We can design small molecule inhibitors to inhibit the phosphorylation of Ser156 or its downstream pathway as a new anticancer therapy. Developing AQP5-specific monoclonal antibody will also be another approach we can take in the future.

\section{AQPs in colorectal cancer}

$\mathrm{AQP1}, \mathrm{AQP3}, \mathrm{AQP} 5$ and AQP9 are found in colorectal cancer. AQP1 had the oncogenic property in colon cancer and was over-expressed in the early stage of the disease and through the late stage of colorectal carcinogenesis [34]. AQP1 expression was correlated with the progression of colon cancer, including lymph node metastasis and lymphovascular invasion. AQP1 was thus proposed as an independent poor prognostic factor in colon cancer [81]. Forced-expressed AQP1 in colon cancer cells increased the plasma membrane water permeability and migration ability, which could be inhibited by AQP1-specific blockers. The mechanism of AQP1-induced cells migration and metastasis in colon cancer cells was the relocalization of actin protein and activation of RhoA and Rac [82]. So downregulation expression of AQP1 in colorectal cancer may be a new therapeutic approach.

The colon cancer had higher expression of AQP3 compared to the normal tissue. The expression intensity of AQP3 was correlated with tumor differentiation and metastasis in colon cancer patients. The migration of colon cancer cells can be inhibited by AQP3-specific inhibitor, as well as EGFR pathway inhibitors [83]. It provided a new therapeutic strategy in colon cancer by blocking AQP3 and EGFR pathway.

AQP5 was over-expressed in colorectal cancer and a strong prognostic biomarker for colorectal cancer. A positive relationship between AQP5 over-expression and the number of circulating tumor cells was observed in colon cancer [84]. Patients with colon cancer expressed with AQP5 had more chances to appear liver metastasis. The over-expression of AQP5 promoted cell proliferation, while deletion of AQP5 inhibited colon cancer cell growth. AQP5 induced tumor proliferation by the activation of Ras-MAPK pathway, cyclin D1/CDK4 complexes, and then phosphorylated retinoblastoma protein in nucleus and caused transcription of genes related with cell proliferation [45].

Besides, AQP5 and AQP9 were associated with the resistance of colon cancer to drugs. Deletion of AQP5 could increase sensitivity to chemotherapeutic drugs by the inhibition of p38 MAPK pathway in colon cancer cells [85]. Down-expression of AQP9 was related with non-responses of colorectal cancer cells to adjuvant chemotherapy [86]. Thus, AQP5-p38 MAPK pathway and AQP9 would be a new therapeutic target to improve drug resistance of colon cancer.

\section{AQPs in hepatic cancer}

Hepatic cancer expresses similar types of AQPs with colon cancer, e.g. AQP1, AQP3, AQP5, and AQP9. AQP1 expression was specially found in cholangiocarcinomas (CCs) and the microvessels of hepatocellular carcinomas (HCCs) while was negative in HCCs and metastatic colorectal carcinomas (MCCs) [87]. The immunohistochemical detection of AQP1 can be more reliable to differentiate CCs from HCCs and MCCs, especially in tumors of which histology alone is not sufficient for a proper diagnosis. AQP1 is thus suggested as the potential diagnostic factor to differentiate diagnosis in liver cancer pathologies.

AQP3 and AQP5 were over-expressed in HCCs and negatively associated with 5 -year disease-free survival and 5-year overall survival. It was suggested that the combination of AQP3 and AQP5 protein expression could be an independent poor prognostic factor for patients with HCCs. Besides, co-expression of AQP3 and AQP5 in HCCs has a significant association with serum AFP and tumor stage and grade. It may be helpful to diagnose HCCs by combining serum AFP and AQP3 and AQP5 [40].

The expression of AQP9 was down-regulated in HCCs and mainly located in non-tumourigenic liver tissue [88]. Deceased AQP9 expression in HCCs can increase resistance of hepatic cancer cells to apoptotic stimulation [53]. It indicated that AQP9 would be a new diagnostic or therapeutic target in hepatic cell cancer.

\section{AQPs in brain cancer}

Astrocytic glioma is the most common primary brain cancer in humans, of which glioblastoma multiforme as 
grade IV glioma is the most malignant primary brain tumor with high mortality and poor prognosis [89]. AQP1, AQP4, and AQP9 are over-expressed in brain tumors and proposed to play an important role in carcinogenesis. AQP1, expressed normally in the choroid plexus epithelium, was over-expressed and up-regulated in all types of glioblastoma and associated with the severity and grades of astrocytoma [13,90,91]. The overexpression of AQP1 was predominantly located in perivascular areas or areas of tumor cell infiltration, distant from the necrotic tumour core [92]. It suggested that AQP1 expression was strongly correlated with tumor angiogenesis and proposed as a therapeutic target in brain cancer. Glioma cells present high rates of aerobic glycolysis, resulting in increased lactic acid production. AQP1 can combine with carbonic anhydrases to shut $\mathrm{H}^{+}$from the intracellular to the extracellular compartment, leading to prevention from tumor cytotoxic edema and acidification of extracellular compartment. The acid extracellular environment promoted glioma cells to release cathepsin B, which increased the invasion of glioma cells [93] (Figure 4).

AQP4 expression was up-regulated and redistributed in gliomablast accompanied by a loss of its polarized expression pattern, and played a role in the brain edema formation [15]. AQP4 over-expression in human astrocytoma was associated with the presence of brain edema detected by the magnetic resonance imaging [2]. The gilomablast was characterized with peritumoral edema and considered as vasogenic brain edema [94]. The redistribution of AQP4 in glioblastoma cells was explained as a reaction to VEGF-induced vasogenic edema in order to facilitate the reabsorption of excessive fluid [95]. It remained to be clarified whether increased expression of AQP4 enhanced edema formation or clearance. AQP4 deletion reduced glioma cell migration and impaired the polymerization of $\mathrm{F}$-actin which is involved in cell-cell adhesion and movement [96]. It was suggested that AQP4 was involved in the control of glioblastoma cell migration and invasion through cytoskeleton rearrangement and cell adhesion regulation [97]. AQP4 was also proposed as an anti-apoptosis target for therapy of glioblastom, evidenced by the finding that siRNA-mediated down-regulation of AQP4 induced glioblastoma cell apoptosis [98].

The over-expression of AQP9 was observed across the whole surface of human glioma cells, associated with the energy metabolism, and counteracted the

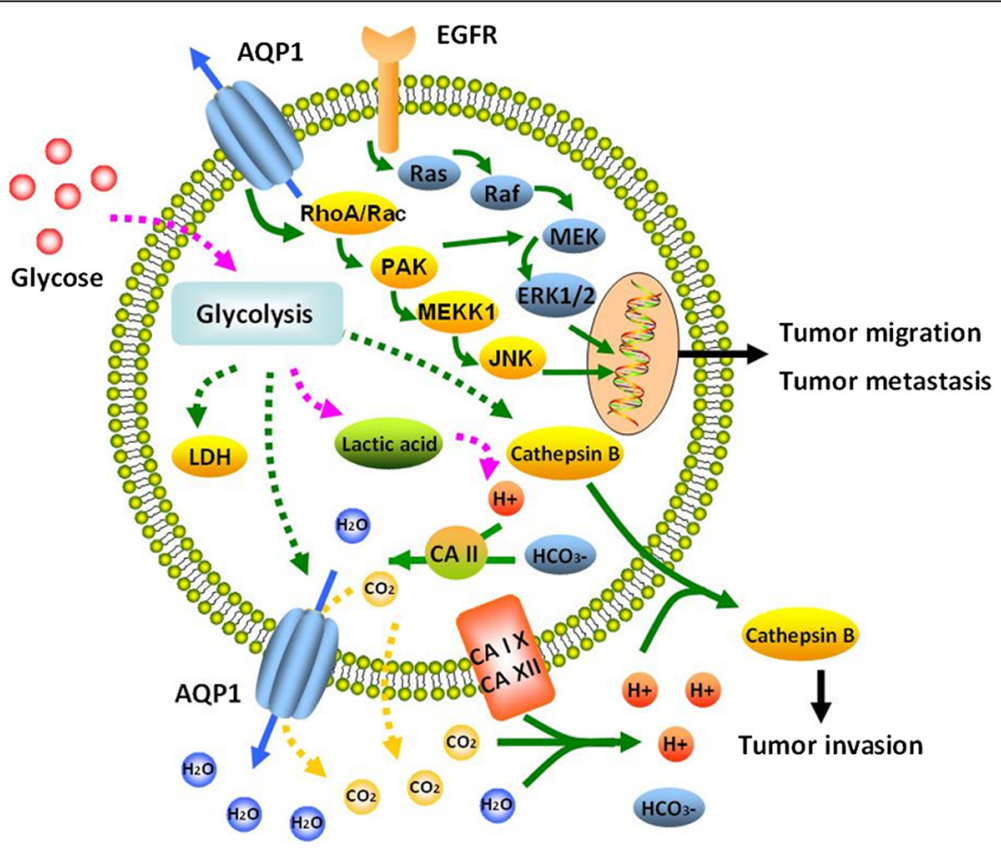

Figure 4 Proposed model of novel role of AQP1 in tumor biology. Tumor cells increase glycose consumption to produce lactic acid, which results in excess $\mathrm{H}^{+}$production and intracellular acidosis. The increase in glycolytic intermediates may up-regulate AQP1, LDH, and cathepsin $B$ through the E-box/ChoRE. Excess $\mathrm{H}^{+}$and $\mathrm{HCO}_{3}^{-}$are catalyzed by intracellular $\mathrm{CAll}$ to produce $\mathrm{H}_{2} \mathrm{O}$ and $\mathrm{CO}_{2}$. The reaction-generated $\mathrm{H}_{2} \mathrm{O}$ is transported to extracellular space to aviod cytotoxic edema by up-regluated $\mathrm{AQP1}$. $\mathrm{CO}_{2}$ may or may not leave the cells through AQP1. Membrane-bound extracellular CA IX and XII may regenerate $\mathrm{H}^{+}$from extracellular $\mathrm{H}_{2} \mathrm{O}$ and $\mathrm{CO}_{2}$, thus leading to shutting $\mathrm{H}^{+}$from the intracellular to the extracellular space to keep the acidification of the extracellular compartment. The acid extracellular environment promotes cells to release cathepsin $\mathrm{B}$, a proteolytic enzyme involved in tumor invasion. AQP1 can induce the activity of RhoA and Rac to increase tumor migration and metastasis. The detailed role of AQP1 in this pathway need more studies. 
glioma-associated lactic acidosis by clearance of glycerol and lactate from the extracellular space [99]. The overexpression of AQP9 in astrocytic glioma was associated with the pathological grades [51], indicating that AQP9 can be a diagnostic biomarker and therapeutic target for brain cancer therapy.

\section{AQPs in breast cancer}

The expression of AQP1, AQP3, and AQP5 is upregulated in breast cancer. AQP1 was mainly overexpressed in the cytoplasm of breast cancer cells and correlated with poor prognosis of patients with breast cancer as an independent prognostic factor [100,101]. The potential mechanism by which AQP1 was involved in breast tumor growth and metastasis may be that AQP1 induced the development of angiogenesis by stimulating and activating endothelial cells through estrogen receptors in human breast cancer. Estrogen can also induce AQP1 expression by activating estrogenresponse element (ERE) in the promoter of the AQP1 gene, resulting in tubulogenesis of vascular endothelial cells [102].

AQP3 was found as a critical and necessary factor for the migration of human breast cancer cells induced by fibroblast growth factor-2 (FGF-2) [103]. FGF-2 could increase AQP3 expression and cell migration through FGFR-PI3K or FGFR-ERK signaling pathways, which was blocked by the deletion of AQP3. AQP3 expression in breast cancer cells was increased by the stimulation with $5^{\prime}$-deoxy-5-fluoropyrimidine nucleosides ( $5^{\prime}$-DFUR), which was used in the chemotherapy of solid tumors [104]. It was proposed that AQP3 may be a limiting factor in pharmacological effects of $5^{\prime}$-DFUR, since the deletion of AQP3 reduced the efficacy of the drug. It seemed that AQP3 can be considered as a chemotherapeutic target to develop the combining strategy for cancer treatment. However, it should be questioned whether AQP3 can act as a breast cancer-specific diagnostic biomarker or therapeutic target.

AQP5 was mainly expressed at apical domains of ductal epithelial cells in breast benign tumor, while such apical polarity of AQP5 in ducts was lost in invasive ductal carcinoma [105]. AQP5 was suggested to regulate the proliferation and migration of breast cancer cells and indicate the prognosis for the patients, probably associated with the estrogen receptor/progesterone receptor or epidermal growth factor receptor 2 [106]. AQP5 over-expression was proposed as an independent prognostic marker of survival for breast cancer patients with estrogen-positive tumor.

\section{Therapeutic targets}

AQPs has been identified in different cancers and associated with tumor proliferation, metastasis, angiogenesis, tumor cell adhesion and tumor-associated edema. Thus, it will be an attractive way for us to make AQPs as the therapeutic targets in anticancer treatment. Now, AQPstarget inhibitors including cysteine-reactive heavy metal-based inhibitors, small-molecule inhibitors which inhibit AQPs expression or AQP-induced water permeation, and monoclonal AQP-specific antibody, have been developed and verified. It is also inspiring that the AQP gene transfer has been developed and applied to clinical therapy that eleven participants with previously irradiated parotid glands after radiation therapy for head or neck cancer received AQP1-cDNA transfer therapy in the Phase I clinical trial [107]. However, the efficacy and safety of AQP gene transfer need more studies.

As mentioned above, mercury can block the watertransport function in AQPs with Cys 189, such as AQP1. Similarly, heavy metal-based AQPs inhibitors such as silver-, gold- or ruthenium-containing compounds were considered as potential anticancer drugs $[108,109]$. For example, the common chemotherapy drug cisplatin inhibited the expression of AQP5 which is up-expressed in ovarian tumor and associated with lymph node metastasis and ascites [110]. There are also several small-molecule compounds without heavy mental, which can block AQPs to inhibit tumor biological functions. Acetazolamide, carbonic anhydrase inhibitor, was found to inhibit the expression of AQP1 which protected tumor from cytotoxic edema by maintenance of extracellular acidification and promoted tumor metastasis in glioma [111]. Besides, several AQP1-target inhibitors including cyclophosphamide, topiramate and anesthetic drugs [112], AQP4-target inhibitors including anti-epileptic drugs, bumetanide, sumatriptan and thiadiazole [9], and AQPs-target inhibitors including $\mathrm{TEA}^{+}$[113] were reported in succession. However, the following studies failed to verify AQPs inhibition of these small-molecule AQPs-target inhibitors by different assays [113].

The AQP4-IgG detected in patients with NMO is specially bound to AQP4 and inhibits AQP4 water permeability, thereby leading to complement-dependent cytotoxicity in astrocytes [114]. Now, the monoclonal AQP4-specific antibody has been developed [115]. Thus, the speculation that AQP4-specific antibody linked with toxin can be used to selectively damage AQP4-expressing glioblastoma cells has been proposed [9]. It is still full of surprise and trouble in the way for developing AQPs-target inhibitors as potential anticancer drugs.

\section{Conclusion and perspective}

Clinical and preclinical studies evidence that the expression of AQPs increase in a number of cancers. Biological 


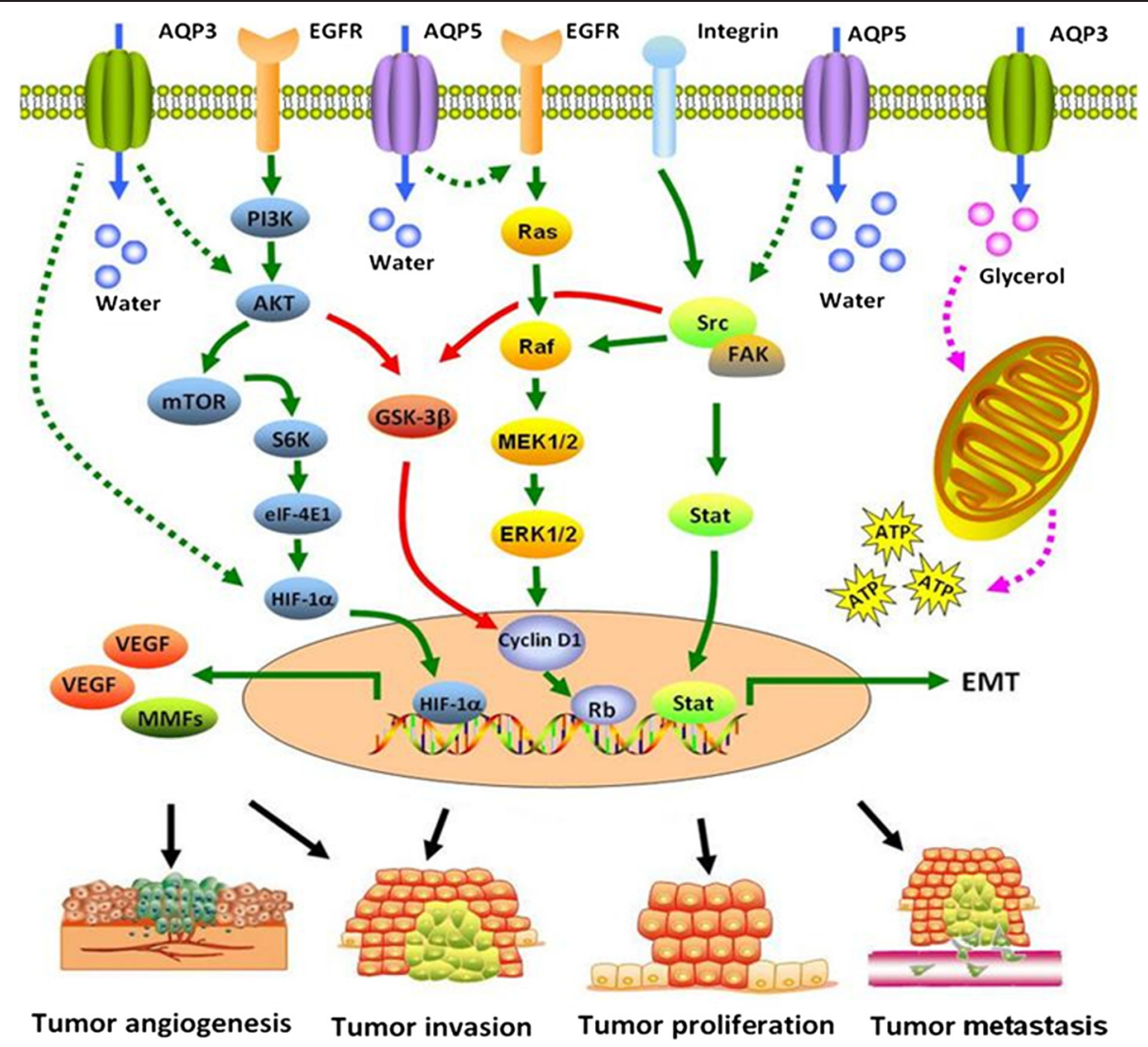

Figure 5 Proposed model of novel roles of AQP3 and AQP5 in tumor biology. AQP5 are exclusively selective for water while AQP3 can transport water and other small neutral solutes such as glycerol. AQP3 increases intracellular glycerol content which is transported to mitochondria to form ATP. Mitochondrial ATP formation provides energy for tumor cell proliferation. AQP3 can directly or indirectly reduce the natural degradation of HIF-1a/ HIF-2a to increase the expression of vascular endothelial growth factor (VEGF), which is a critical regulator in tumor angiogenesis and vessel maturation. AQP3 may directly or indirectly activate AKT to increase MMPs, resulting in tumor invasion. AQP5 induces activation of the epidermal growth factor receptor (EGFR), extracellular receptor kinase (ERK1/2) pathway to faciliate tumor cell proliferation and metastasis. In addition, AQP5 is phosphorylated on Ser156 and bind the the SH3 domain of Src to promote EMT activity in tumor cells. The roles of AQP3 and AQP5 in these pathways are not fully elucidated and still need further exploration.

functions and singling pathways of AQPs in cancer are intensively investigated in condition of AQP deletion (Figure 5). AQPs play an important role in brain water homeostasis, exocrine gland secretion, urine concentration, skin moisturization, fat metabolism or neural signal transduction. AQPs are also involved in the carcinogenesis and pathogenesis of tumor-associated edema, tumor cell proliferation and migration, or tumor angiogenesis. The results from studies in vivo or vitro also show the attractive opportunities for AQPs-target therapy. AQPs-target inhibitors and AQPs-specific monoclonal antibody, even AQP gene transfer, are developed to provide new therapeutic strategy in anticancer treatment. However, there is still a long way to clearly elucidate the specificity of AQPs in the pathogenesis, metabolisms, and controls of various cancers, or resistance and tolerance to anticancer therapies. The process in AQPs-target inhibitors development has also been slow and none AQPassociated drugs has been applied for clinical anticancer therapy by far.

\section{Abbreviations}

AQPS: Aquaporins; ADC: Adenocarcinoma; AVP: Vasopressin; BAC: Bronchoalveolar carcinoma; BBB: Blood-brain barrier; CCs: Cholangiocarcinomas; CML: Chronic myelogenous leukemia; CNS: Central nervous system; EGFR: Epidermal growth factor receptor; ERE: Estrogen-response element; ERK: Extracellular receptor kinase; FGF-2: Fibroblast growth factor-2; HCCs: Hepatocellular carcinomas; MCCs: Metastatic colorectal carcinomas; NMO: Neuromyelitis optica; NPA: Asparagine-proline-alanine; NSCLC: Non-small cell carcinoma; OAPs: Orthogonal arrays of particles; PKA: CAMP-protein kinase; VEGF: Vascular endothelial growth factor; 5'-DFUR: 5'-deoxy-5-fluoropyrimidine nucleosides.

\section{Competing interests}

The authors declare that they have no competing interests. 


\section{Authors' contributions}

JW, LF and ZTZ wrote the manuscript and drafted the figures. $\mathrm{MHZ}$ and DW searched and analyzed the publication data. ZHC and HZS provided the original idea and critically revised the manuscript and figures. All authors read and approved the final manuscript.

\section{Author details}

${ }^{1}$ Department of Pulmonary Medicine, Zhongshan Hospital, Fudan University, Shanghai, China. ${ }^{2}$ Minghang Hospital, Fudan University, Shanghai, China.

${ }^{3}$ The First Hospital of Liaoning Medical University, Jingzhou, China.

\section{Received: 3 January 2015 Accepted: 17 February 2015}

\section{Published online: 21 March 2015}

\section{References}

1. Verkman AS. More than just water channels: unexpected cellular roles of aquaporins. J Cell Sci. 2005;118:3225-32.

2. Nico B, Ribatti D. Role of aquaporins in cell migration and edema formation in human brain tumors. Exp Cell Res. 2011;317:2391-6.

3. Herrera M, Garvin JL. Aquaporins as gas channels. Pflugers Arch. 2011:462:623-30

4. Musa-Aziz R, Chen LM, Pelletier MF, Boron WF. Relative CO2/NH3 selectivities of AQP1, AQP4, AQP5, AmtB, and RhAG. Proc Natl Acad Sci U S A. 2009;106:5406-11.

5. Yu J, Yool AJ, Schulten K, Tajkhorshid E. Mechanism of gating and ion conductivity of a possible tetrameric pore in aquaporin-1. Structure. 2006;14:1411-23.

6. Badaut J, Fukuda AM, Jullienne A, Petry KG. Aquaporin and brain diseases. Biochim Biophys Acta. 1840;2014:1554-65.

7. Cartagena CM, Phillips KL, Tortella FC, Dave JR, Schmid KE. Temporal alterations in aquaporin and transcription factor HIF1alpha expression following penetrating ballistic-like brain injury (PBBI). Mol Cell Neurosci. 2014:60:81-7.

8. Strand L, Moe SE, Solbu TT, Vaadal M, Holen T. Roles of aquaporin-4 isoforms and amino acids in square array assembly. Biochemistry-Us. 2009;48:5785-93.

9. Papadopoulos MC, Saadoun S. Key roles of aquaporins in tumor biology. Biochim Biophys Acta. 2014.

10. Jin BJ, Rossi A, Verkman AS. Model of aquaporin-4 supramolecular assembly in orthogonal arrays based on heterotetrameric association of M1-M23 isoforms. Biophys J. 2011;100:2936-45.

11. Nicchia GP, Mastrototaro M, Rossi A, Pisani F, Tortorella C, Ruggieri M, et al. Aquaporin-4 orthogonal arrays of particles are the target for neuromyelitis optica autoantibodies. Glia. 2009;57:1363-73.

12. Guan G, Dong Z, Sun K. Correlation between the expression of aquaporin and the micro-angiogenesis in laryngeal carcinoma. Lin Chung Er Bi Yan Hou Tou Jing Wai Ke Za Zhi. 2009;23:219-21.

13. Saadoun S, Papadopoulos MC, Davies DC, Bell BA, Krishna S. Increased aquaporin 1 water channel expression in human brain tumours. $\mathrm{Br} \mathrm{J}$ Cancer. 2002;87:621-3

14. Saadoun S, Papadopoulos MC, Davies DC, Krishna S, Bell BA. Aquaporin-4 expression is increased in oedematous human brain tumours. J Neurol Neurosurg Psychiatry. 2002;72:262-5.

15. Warth A, Simon P, Capper D, Goeppert B, Tabatabai G, Herzog H, et al. Expression pattern of the water channel aquaporin- 4 in human gliomas is associated with blood-brain barrier disturbance but not with patient survival. J Neurosci Res. 2007:85:1336-46.

16. Hoque MO, Soria JC, Woo J, Lee T, Lee J, Jang SJ, et al. Aquaporin 1 is overexpressed in lung cancer and stimulates $\mathrm{NIH}-3 \mathrm{~T} 3$ cell proliferation and anchorage-independent growth. Am J Pathol. 2006;168:1345-53.

17. Sales AD, Lobo CH, Carvalho AA, Moura AA, Rodrigues AP. Structure, function, and localization of aquaporins: their possible implications on gamete cryopreservation. Genet Mol Res. 2013;12:6718-32.

18. Fujiyoshi Y, Mitsuoka K, de Groot BL, Philippsen A, Grubmuller H, Agre P, et al, Structure and function of water channels. Curr Opin Struct Biol. 2002;12:509-15.

19. Preston GM, Jung JS, Guggino WB, Agre P. The mercury-sensitive residue at cysteine 189 in the CHIP28 water channel. J Biol Chem. 1993;268:17-20.

20. Verkman AS, Mitra AK. Structure and function of aquaporin water channels. Am J Physiol Renal Physiol. 2000;278:F13-28.

21. Jensen MO, Tajkhorshid E, Schulten K. Electrostatic tuning of permeation and selectivity in aquaporin water channels. Biophys J. 2003;85:2884-99.
22. Jung JS, Preston GM, Smith BL, Guggino WB, Agre P. Molecular structure of the water channel through aquaporin CHIP. The hourglass model. J Biol Chem. 1994;269:14648-54.

23. Jensen MO, Tajkhorshid E, Schulten $\mathrm{K}$. The mechanism of glycerol conduction in aquaglyceroporins. Structure. 2001;9:1083-93.

24. Skelton LA, Boron WF, Zhou Y. Acid-base transport by the renal proximal tubule. J Nephrol. 2010;23 Suppl 16:S4-18.

25. $\mathrm{Xu} \mathrm{L}$, Zhou $\mathrm{Y}$, Boron WF. Role of aquaporin 1 (AQP1) as a CO2 channel in chronic metabolic acidosis. Faseb J. 2011;834:1.

26. Herrera M, Garvin JL. Novel role of AQP-1 in NO-dependent vasorelaxation. Am J Physiol Renal Physiol. 2007;292:F1443-51.

27. Gannon BJ, Warnes GM, Carati CJ, Verco CJ. Aquaporin-1 expression in visceral smooth muscle cells of female rat reproductive tract. J Smooth Muscle Res. 2000;36:155-67.

28. Verkman AS. Aquaporins in clinical medicine. Annu Rev Med. 2012;63:303-16.

29. Kumari SS, Varadaraj K. Aquaporin 0 plays a pivotal role in refractive index gradient development in mammalian eye lens to prevent spherical aberration. Biochem Biophys Res Commun. 2014;452:986-91.

30. Deen PM, Verdijk MA, Knoers NV, Wieringa $B$, Monnens LA, van Os $C H$, et al. Requirement of human renal water channel aquaporin-2 for vasopressin-dependent concentration of urine. Science. 1994;264:92-5.

31. Nielsen S, Frokiaer J, Marples D, Kwon TH, Agre P, Knepper MA. Aquaporin in the kidney: from molecules to medicine. Physiol Rev. 2002;82:205-44.

32. Papadopoulos MC, Verkman AS. Aquaporin water channels in the nervous system. Nat Rev Neurosci. 2013;14:265-77.

33. Gomes D, Agasse A, Thiebaud P, Delrot S, Geros H, Chaumont F. Aquaporins are multifunctional water and solute transporters highly divergent in living organisms. Biochim Biophys Acta. 2009;1788:1213-28.

34. Moon C, Soria JC, Jang SJ, Lee J, Obaidul HM, Sibony M, et al. Involvement of aquaporins in colorectal carcinogenesis. Oncogene. 2003;22:6699-703.

35. Yin T, Yu S, Xiao L, Zhang J, Liu C, Lu Y, et al. Correlation between the expression of aquaporin 1 and hypoxia-inducible factor 1 in breast cancer tissues. J Huazhong Univ Sci Technolog Med Sci. 2008;28:346-8.

36. Mobasheri A, Airley R, Hewitt SM, Marples D. Heterogeneous expression of the aquaporin 1 (AQP1) water channel in tumors of the prostate, breast, ovary, colon and lung: a study using high density multiple human tumor tissue microarrays. Int J Oncol. 2005;26:1149-58.

37. Yang JH, Shi YF, Chen XD, Qi WJ. The influence of aquaporin-1 and microvessel density on ovarian carcinogenesis and ascites formation. Int J Gynecol Cancer. 2006;16 Suppl 1:400-5.

38. Hara-Chikuma M, Verkman AS. Prevention of skin tumorigenesis and impairment of epidermal cell proliferation by targeted aquaporin-3 gene disruption. Mol Cell Biol. 2008;28:326-32.

39. Liu S, Zhang $S$, Jiang $H$, Yang $Y$, Jiang $Y$. Co-expression of AQP3 and AQP5 in esophageal squamous cell carcinoma correlates with aggressive tumor progression and poor prognosis. Med Oncol. 2013;30:636

40. Guo X, Sun T, Yang M, Li Z, Li Z, Gao Y. Prognostic value of combined aquaporin 3 and aquaporin 5 overexpression in hepatocellular carcinoma. Biomed Res Int. 2013:2013:206525.

41. Kafe H, Verbavatz JM, Cochand-Priollet B, Castagnet P, Vieillefond A. Collecting duct carcinoma: an entity to be redefined? Virchows Arch. 2004;445:637-40

42. Kusayama M, Wada K, Nagata M, Ishimoto S, Takahashi H, Yoneda M, et al. Critical role of aquaporin 3 on growth of human esophageal and oral squamous cell carcinoma. Cancer Sci. 2011;102:1128-36.

43. Xie $Y$, Wen $X$, Jiang $Z$, Fu HQ, Han H, Dai L. Aquaporin 1 and aquaporin 4 are involved in invasion of lung cancer cells. Clin Lab. 2012:58:75-80.

44. Niu D, Kondo T, Nakazawa T, Kawasaki T, Yamane T, Mochizuki K, et al. Differential expression of aquaporins and its diagnostic utility in thyroid cancer. PLoS One. 2012;7:e40770.

45. Kang SK, Chae YK, Woo J, Kim MS, Park JC, Lee J, et al. Role of human aquaporin 5 in colorectal carcinogenesis. Am J Pathol. 2008;173:518-25.

46. Chae YK, Kang SK, Kim MS, Woo J, Lee J, Chang S, et al. Human AQP5 plays a role in the progression of chronic myelogenous leukemia (CML). PLoS One. 2008;3:e2594.

47. Zhang Z, Chen Z, Song Y, Zhang P, Hu J, Bai C. Expression of aquaporin 5 increases proliferation and metastasis potential of lung cancer. J Pathol. 2010;221:210-20.

48. Yan C, Yang J, Shen L, Chen X. Inhibitory effect of Epigallocatechin gallate on ovarian cancer cell proliferation associated with aquaporin 5 expression. Arch Gynecol Obstet. 2012;285:459-67. 
49. Watanabe T, Fujii T, Oya T, Horikawa N, Tabuchi Y, Takahashi Y, et al. Involvement of aquaporin-5 in differentiation of human gastric cancer cells. J Physiol Sci. 2009;59:113-22.

50. Lacroix L, Lazar V, Michiels S, Ripoche H, Dessen P, Talbot M, et al. Follicular thyroid tumors with the PAX8-PPARgamma1 rearrangement display characteristic genetic alterations. Am J Pathol. 2005;167:223-31.

51. Tan G, Sun SQ, Yuan DL. Expression of the water channel protein aquaporin-9 in human astrocytic tumours: correlation with pathological grade. J Int Med Res. 2008;36:777-82.

52. Yang JH, Yan CX, Chen XJ, Zhu YS. Expression of aquaglyceroporins in epithelial ovarian tumours and their clinical significance. J Int Med Res 2011;39:702-11.

53. Jablonski EM, Mattocks MA, Sokolov E, Koniaris LG, Hughes FJ, Fausto N, et al. Decreased aquaporin expression leads to increased resistance to apoptosis in hepatocellular carcinoma. Cancer Lett. 2007;250:36-46.

54. Wang W, Li Q, Yang T, Bai G, Li D, Li Q, et al. Expression of AQP5 and AQP8 in human colorectal carcinoma and their clinical significance. World J Surg Oncol. 2012;10:242

55. Solenov E, Watanabe H, Manley GT, Verkman AS. Sevenfold-reduced osmotic water permeability in primary astrocyte cultures from AQP-4-deficient mice, measured by a fluorescence quenching method. Am J Physiol Cell Physiol. 2004;286:C426-32.

56. Papadopoulos MC, Verkman AS. Aquaporin-4 gene disruption in mice reduces brain swelling and mortality in pneumococcal meningitis. J Biol Chem. 2005;280:13906-12.

57. Katada R, Akdemir G, Asavapanumas N, Ratelade J, Zhang H, Verkman AS. Greatly improved survival and neuroprotection in aquaporin-4-knockout mice following global cerebral ischemia. Faseb J. 2014;28:705-14.

58. Delporte C. Aquaporins in salivary glands and pancreas. Biochim Biophys Acta. 1840;2014:1524-32.

59. Krane CM, Melvin JE, Nguyen HV, Richardson L, Towne JE, Doetschman T, et al. Salivary acinar cells from aquaporin 5-deficient mice have decreased membrane water permeability and altered cell volume regulation. J Biol Chem. 2001;276:23413-20.

60. Matsuzaki T, Susa T, Shimizu K, Sawai N, Suzuki T, Aoki T, et al. Function of the membrane water channel aquaporin-5 in the salivary gland. Acta Histochem Cytochem. 2012;45:251-9.

61. Kortenoeven ML, Fenton RA. Renal aquaporins and water balance disorders. Biochim Biophys Acta. 1840;2014:1533-49.

62. King LS, Choi M, Fernandez PC, Cartron JP, Agre P. Defective urinaryconcentrating ability due to a complete deficiency of aquaporin-1. N Engl J Med. 2001;345:175-9.

63. Rojek A, Fuchtbauer EM, Kwon TH, Frokiaer J, Nielsen S. Severe urinary concentrating defect in renal collecting duct-selective AQP2 conditional-knockout mice. Proc Natl Acad Sci U S A. 2006;103:6037-42.

64. Hara-Chikuma M, Verkman AS. Roles of aquaporin-3 in the epidermis J Invest Dermatol. 2008;128:2145-51.

65. Lee Y, Je YJ, Lee SS, Li ZJ, Choi DK, Kwon YB, et al. Changes in transepidermal water loss and skin hydration according to expression of aquaporin-3 in psoriasis. Ann Dermatol. 2012;24:168-74.

66. Hara M, Ma T, Verkman AS. Selectively reduced glycerol in skin of aquaporin-3-deficient mice may account for impaired skin hydration, elasticity, and barrier recovery. J Biol Chem. 2002;277:46616-21.

67. Hara-Chikuma M, Sohara E, Rai T, Ikawa M, Okabe M, Sasaki S, et al. Progressive adipocyte hypertrophy in aquaporin-7-deficient mice: adipocyte glycerol permeability as a novel regulator of fat accumulation. J Biol Chem. 2005;280:15493-6.

68. Saadoun S, Papadopoulos MC, Hara-Chikuma M, Verkman AS. Impairment of angiogenesis and cell migration by targeted aquaporin-1 gene disruption. Nature. 2005:434:786-92.

69. Hu J, Verkman AS. Increased migration and metastatic potential of tumor cells expressing aquaporin water channels. Faseb J. 2006;20:1892-4.

70. Auguste KI, Jin S, Uchida K, Yan D, Manley GT, Papadopoulos MC, et al. Greatly impaired migration of implanted aquaporin-4-deficient astroglial cells in mouse brain toward a site of injury. Faseb J. 2007;21:108-16.

71. Saadoun S, Papadopoulos MC, Watanabe H, Yan D, Manley GT, Verkman AS. Involvement of aquaporin-4 in astroglial cell migration and glial scar formation. J Cell Sci. 2005;118:5691-8.

72. Hiroaki Y, Tani K, Kamegawa A, Gyobu N, Nishikawa K, Suzuki H, et al. Implications of the aquaporin-4 structure on array formation and cell adhesion. J Mol Biol. 2006;355:628-39.
73. Gonen T, Sliz P, Kistler J, Cheng Y, Walz T. Aquaporin-0 membrane junctions reveal the structure of a closed water pore. Nature. 2004:429:193-7.

74. Warth A, Muley T, Meister M, Herpel E, Pathil A, Hoffmann H, et al. Loss of aquaporin-4 expression and putative function in non-small cell lung cancer. BMC Cancer. 2011;11:161.

75. Machida Y, Ueda Y, Shimasaki M, Sato K, Sagawa M, Katsuda S, et al. Relationship of aquaporin 1, 3, and 5 expression in lung cancer cells to cellular differentiation, invasive growth, and metastasis potential. Hum Pathol. 2011:42:669-78.

76. Lopez-Campos JL, Sanchez SR, Gomez IL, Marquez E, Ortega RF, Cejudo P, et al. Overexpression of Aquaporin-1 in lung adenocarcinomas and pleural mesotheliomas. Histol Histopathol. 2011;26:451-9.

77. Liu YL, Matsuzaki T, Nakazawa T, Murata S, Nakamura N, Kondo T, et al. Expression of aquaporin 3 (AQP3) in normal and neoplastic lung tissues. Hum Pathol. 2007:38:171-8.

78. Xia H, Ma YF, Yu CH, Li YJ, Tang J, Li JB, et al. Aquaporin 3 knockdown suppresses tumour growth and angiogenesis in experimental non-small cell lung cancer. Exp Physiol. 2014;99:974-84.

79. Xu JL, Xia R. The emerging role of aquaporin 5 (AQP5) expression in systemic malignancies. Tumour Biol. 2014;35:6191-2.

80. Woo J, Lee J, Chae YK, Kim MS, Baek JH, Park JC, et al. Overexpression of AQP5, a putative oncogene, promotes cell growth and transformation. Cancer Lett. 2008:264:54-62.

81. Yoshida T, Hojo S, Sekine S, Sawada S, Okumura T, Nagata T, et al. Expression of aquaporin-1 is a poor prognostic factor for stage II and III colon cancer. Mol Clin Oncol. 2013;1:953-8.

82. Jiang Y. Aquaporin-1 activity of plasma membrane affects HT20 colon cancer cell migration. lubmb Life. 2009:61:1001-9.

83. Li A, Lu D, Zhang Y, Li J, Fang Y, Li F, et al. Critical role of aquaporin-3 in epidermal growth factor-induced migration of colorectal carcinoma cells and its clinical significance. Oncol Rep. 2013;29:535-40.

84. Shan T, Cui X, Li W, Lin W, Li Y. AQP5: a novel biomarker that predicts poor clinical outcome in colorectal cancer. Oncol Rep. 2014;32:1564-70.

85. Shi $X$, Wu S, Yang $Y$, Tang L, Wang Y, Dong J, et al. AQP5 silencing suppresses p38 MAPK signaling and improves drug resistance in colon cancer cells. Tumour Biol. 2014;35:7035-45.

86. Dou R, Deng Y, Huang L, Fu S, Tan S, Wang L, et al. Multi-microarray identifies lower AQP9 expression in adjuvant chemotherapy nonresponders with stage III colorectal cancer. Cancer Lett. 2013;336:106-13.

87. Mazal PR, Susani M, Wrba F, Haitel A. Diagnostic significance of aquaporin-1 in liver tumors. Hum Pathol. 2005;36:1226-31.

88. Padma S, Smeltz AM, Banks PM, lannitti DA, McKillop IH. Altered aquaporin 9 expression and localization in human hepatocellular carcinoma. HPB (Oxford). 2009;11:66-74

89. Altieri R, Agnoletti A, Quattrucci F, Garbossa D, Calamo SF, Bozzaro M, et al. Molecular biology of gliomas: present and future challenges. Transl Med UniSa. 2014;10:29-37.

90. Oshio K, Binder DK, Liang Y, Bollen A, Feuerstein B, Berger MS, et al. Expression of the aquaporin-1 water channel in human glial tumors. Neurosurgery. 2005;56:375-81.

91. Oshio K, Binder DK, Bollen A, Verkman AS, Berger MS, Manley GT. Aquaporin-1 expression in human glial tumors suggests a potential novel therapeutic target for tumor-associated edema. Acta Neurochir Suppl. 2003;86:499-502.

92. El HN, Bankfalvi A, Herring A, Adamzik M, Lambertz N, Zhu Y, et al. Correlation of aquaporin-1 water channel protein expression with tumor angiogenesis in human astrocytoma. Anticancer Res. 2013;33:609-13.

93. Hayashi Y, Edwards NA, Proescholdt MA, Oldfield EH, Merrill MJ. Regulation and function of aquaporin-1 in glioma cells. Neoplasia. 2007:9:777-87.

94. Betz AL, lannotti F, Hoff JT. Brain edema: a classification based on blood-brain barrier integrity. Cerebrovasc Brain Metab Rev. 1989;1:133-54.

95. Yang L, Wang X, Zhen S, Zhang S, Kang D, Lin Z. Aquaporin-4 upregulated expression in glioma tissue is a reaction to glioma-associated edema induced by vascular endothelial growth factor. Oncol Rep. 2012;28:1633-8.

96. Ding T, Ma Y, Li W, Liu X, Ying G, Fu L, et al. Role of aquaporin-4 in the regulation of migration and invasion of human glioma cells. Int J Oncol. 2011;38:1521-31.

97. Ding T, Gu F, Fu L, Ma YJ. Aquaporin-4 in glioma invasion and an analysis of molecular mechanisms. J Clin Neurosci. 2010;17:1359-61.

98. Ding T, Zhou Y, Sun K, Jiang W, Li W, Liu X, et al. Knockdown a water channel protein, aquaporin-4, induced glioblastoma cell apoptosis. PLoS One. 2013;8:e66751. 
99. Warth A, Mittelbronn M, Hulper P, Erdlenbruch B, Wolburg H. Expression of the water channel protein aquaporin-9 in malignant brain tumors. Appl Immunohistochem Mol Morphol. 2007;15:193-8.

100. Zhang B, Liu FF, Ma YJ, Gu F. Cytoplasmic expression of aquaporin-1 in breast cancer cells and its relationship with clinicopathological characteristics and prognosis. Zhonghua Zhong Liu Za Zhi. 2013:35:904-9.

101. Otterbach F, Callies R, Adamzik M, Kimmig R, Siffert W, Schmid KW, et al. Aquaporin 1 (AQP1) expression is a novel characteristic feature of a particularly aggressive subgroup of basal-like breast carcinomas. Breast Cancer Res Treat. 2010;120:67-76.

102. Zou LB, Shi S, Zhang RJ, Wang TT, Tan YJ, Zhang D, et al. Aquaporin-1 plays a crucial role in estrogen-induced tubulogenesis of vascular endothelial cells. J Clin Endocrinol Metab. 2013:98:E672-82.

103. Cao XC, Zhang WR, Cao WF, Liu BW, Zhang F, Zhao HM, et al. Aquaporin3 is required for FGF-2-induced migration of human breast cancers. PLoS One. 2013;8:e56735

104. Triqueros-Motos L, Perez-Torras S, Casado FJ, Molina-Arcas M Pastor-Anglada M. Aquaporin 3 (AQP3) participates in the cytotoxic response to nucleoside-derived drugs. BMC Cancer. 2012;12:434.

105. Jung HJ, Park JY, Jeon HS, Kwon TH. Aquaporin-5: a marker protein for proliferation and migration of human breast cancer cells. PLoS One. 2011;6:e28492

106. Lee SJ, Chae YS, Kim JG, Kim WW, Jung JH, Park HY, et al. AQP5 expression predicts survival in patients with early breast cancer. Ann Surg Oncol. 2014;21:375-83.

107. Baum BJ, Alevizos I, Zheng C, Cotrim AP, Liu S, McCullagh L, et al. Early responses to adenoviral-mediated transfer of the aquaporin-1 CDNA for radiation-induced salivary hypofunction. Proc Natl Acad Sci U S A 2012;109:19403-7.

108. Bergamo A, Sava G. Ruthenium anticancer compounds: myths and realities of the emerging metal-based drugs. Dalton Trans. 2011;40:7817-23.

109. Niemietz CM, Tyerman SD. New potent inhibitors of aquaporins: silver and gold compounds inhibit aquaporins of plant and human origin. Febs Lett. 2002;531:443-7.

110. Yang J, Yan C, Zheng W, Chen X. Proliferation inhibition of cisplatin and aquaporin 5 expression in human ovarian cancer cell CAOV3. Arch Gynecol Obstet. 2012:285:239-45.

111. Pedersen SF, Hoffmann EK, Mills JW. The cytoskeleton and cell volume regulation. Comp Biochem Physiol A Mol Integr Physiol. 2001;130:385-99.

112. Monzani E, Shtil AA, La Porta CA. The water channels, new druggable targets to combat cancer cell survival, invasiveness and metastasis. Curr Drug Targets. 2007;8:1132-7.

113. Verkman AS, Anderson MO, Papadopoulos MC. Aquaporins: important but elusive drug targets. Nat Rev Drug Discov. 2014;13:259-77.

114. Papadopoulos MC, Verkman AS. Aquaporin 4 and neuromyelitis optica. Lancet Neurol. 2012:11:535-44.

115. Tradtrantip L, Zhang H, Saadoun S, Phuan PW, Lam C, Papadopoulos MC, et al. Anti-aquaporin-4 monoclonal antibody blocker therapy for neuromyelitis optica. Ann Neurol. 2012;71:314-22.

\section{Submit your next manuscript to BioMed Central and take full advantage of:}

- Convenient online submission

- Thorough peer review

- No space constraints or color figure charges

- Immediate publication on acceptance

- Inclusion in PubMed, CAS, Scopus and Google Scholar

- Research which is freely available for redistribution 